\title{
Interactive comment on "Modeling macropore seepage fluxes from soil water content time series by inversion of a dual permeability model" by Nicolas Dalla Valle et al.
}

\section{Anonymous Referee \#2}

Received and published: 17 July 2017

The paper presents a dual permeability (DPM) simplified 1D model by combining a Richard's type of solution for the matrix flow and a Kinematic wave solution for the macropore domain. The simplification allows to reduce the number of parameters which allows an easier implementation of an inverse modeling subroutine. The model was calibrated and the uncertainty of input and output parameters were both estimated with the FME package for $\mathrm{R}$.

The reduction idea plus the combination of mathematical 1D alternative formulation of DPM, validation with lab experimental measurements and the quantification of input and output uncertainty makes a complete approach to the problem.

Printer-friendly version

Discussion paper 
The model simplification and input reduction is always desired for models as their overparametrization makes their implementation difficult and it may even increase their uncertainty. Therefore, the intention of reducing the number of parameters by simplifying the model from a mathematical point of view is the strong selling point of this study.

However, the study points out that their aim is divided in two main parts; Developing the low complexity model and develop a model inversion scheme. For the former one, it is not clear to the reader or reviewer if lower complexity model is desirable as even the authors point out that the model can only be implemented for the specific case of the controlled conditions in the lab, the obtained results are not very good, they are only tested for one infiltration event of the 15 intended and last but not least, it reduces the flexibility for fitting the capacity of retention functions.

For the latter part, it seems that the necessity of the inversion modeling process for the calibration is not sufficiently explained and seems merely a product of the availability of a software package. This last comment doesn't mean it cannot be done but it shouldn't be one of the main goals of the study as all models in any field require calibration and validation. If such a robust implementation is really required, the study should also probe it by comparing the results of different calibration methods so that the expected drawbacks (equability and local minima) are proven to exist.

If performing all these additions and addressing the comments for improving the paper, I will accept the paper with major changes.

Regarding the model development section:

Please explicitly include the reduction of parameters (which parameters are required if no simplification is done (presented Table 2) vs which parameter are required in the proposed model) with respect to the different methods so that the study becomes more attractive to the reader.

It is important to clarify that the matric water diffusivity referred in the study is a "bor- 
rowed" term to refer to describe the gradient in time of the soil water content (in the differential form of a diffusion process) instead of a diffusive particle solute movement in gases or liquids. Hence it is recommended to use the term Hydraulic diffusivity as explained for example in the book of Hillel "Introduction to environmental Soil Physics".

The deduction of the macropore flux is missing a scheme (i.e. as presented by Hincapié and Germann 2009) in which its deduction and variables are presented for the new proposed model representation.

The exchange lumped term $r$ is a function the same 3 parameters ( $G, d$ and ) which are present in both Dual permeability and dual porosity models described in literature (See Gerke and Van Genuchten 1993, Evaluation of a first order water transfer term for variably saturated dual-porosity flow models). It would be interesting to compare the obtained results with the ones presented in literature as the posterior $r$ distribution seems to reflect a high degree of uncertainty in the model. Furthermore, it would be valuable if this macropore exchange parameters where fixed based on literature and see how much they either reduce or increase the uncertainty bounds which will allow to have a first impression of their importance for the studied soil in the proposed model.

According to the manuscript, 45 time series extracted from 15 rainfall events were measured but only 1 of them presented observable seepage fluxes which might imply that the experiment was not correctly designed. It might be related to the high silt content of the soil probes. Also note that the method presented in Campbel (1974) was validated against sandy loam (higher content of sand than silt).

Regarding the inverse modeling scheme:

It is not explicitly mentioned which are the input parameters which are going to be found by the inverse modeling algorithm for each of the optimization steps.

A very "robust" algorithm for inverse modeling is used for fitting a rather simple model (as pointed by the authors) with a reduced number of input parameters which gives the

Printer-friendly version

Discussion paper 
impression that its implementation might not be required. Two assumptions are pointed to support the use of the algorithm (e.g existence of local minimums and equifinality. For the former one, it must be proved that this is the case by implementing a simpler algorithm in which local minima is obtained. It is expected that with monotonous and continuous functions, the chances of finding local minima for the objective functions presented are reduced. For the latter one, it is expected that this is still the case as too many parameters (e.g. for the first inversion a,b, $n$ and L,r,c and theta_s) are fitted from basically two measurements of soil water content $(5 \mathrm{~cm}$ and $12 \mathrm{~cm})$. All water retention functions are not linear which implies that at least 3 different measurements should be used for a reliable fitting

The uncertainty weights ( $w 1$ and w2) used inside the objective functions for minimization are chosen arbitrarily. Please explain the choices or even if possible, make a small sensitivity test as they can bias the obtained distributions for the unknown parameters.

The authors imply that a double optimization inverse modeling framework is needed to avoid equifinality. So the questions that arise are: why are the authors want to avoid equifinality while including more than one water retention function as part of the study (Note that equifinality also implies that different models may perform similarly) and is it important to avoid it when the parameter results are presented as uncertainties for each parameter?

It will be very valuable to compare parameters obtained from the initial inverse modeling with respect to the same ones obtained in the next inverse modeling. Further more, if one of the main goals of the manuscript is to show the importance of the whole inversion method (Besides the simplification of the model as pointed in page 3 , line 20), a comparison against a single inverse modeling optimization of all parameters will benefit the study significantly. Otherwise, what is the point of doing 2 step optimization without probing the effects of equifinality or local minima encountering ?

As a suggestion: For this kind of studies, the GLUE (Beven and Binley) methodology

Printer-friendly version

Discussion paper
Interactive comment 
may be a better option as it accepts the fact that equifinality is present for all paramters and models and hence evaluates the models based on a likelihood function.

Results and discussion:

In figure 3, the range of measurements differs from the ones obtained for the estimated functions. If these curves were used as seed values for the next optimization, would it imply that the measurements obtained at $-5 \mathrm{~cm}$ are useless? Otherwise explain the implication of not having the fitted functions inside the measured bounds. Moreover, is it necessary to perform the initial model inversion? Ideally this step will avoid the entrapment on local minima but evidence of such value must be supported, especially for the fitting on monotonic functions such as the ones presented in this manuscript.

In figures 3 and 4 , it is not clear how the $90 \%$ confidence intervals where obtained and they are almost non-visible, especially in figure 4.

In Figure 4, it is also not clear why the dashed line (Shiozawa) and long dashed line (Oosterveld and Chang) cut the horizontal axis between 0.2 and 0.3 theta values. Whereas the predicted values may go as far as 1.0.

In section 6.1 it is stated that there was a significant reduction of uncertainty after the second model inversion step but it is also acknowledged that data from the first optimization is not shown. Why? What would be the result with one general inversion modeling of all the parameters at the same time?

In the last paragraph of the conclusions it is stated that the prediction of the water content time series at $12 \mathrm{~cm}$ is somewhat worse than the prediction at $5 \mathrm{~cm}$. However, the parameter estimation is better fitted for the measurements at $12 \mathrm{~cm}$ than the ones obtained at $5 \mathrm{~cm}$ which also are said to be close to the global optimum (see Figure 3 ). But appreciations would mean that the author is either getting good results at $5 \mathrm{~cm}$ with worst fitting values at this depth and is getting bad results in the moisture content time series at $12 \mathrm{~cm}$ while having a good fit of the retention function at that depth. This

Printer-friendly version

Discussion paper 
may be a good evidence that one inverse modeling procedure of all parameters at the same time might be better (but more complicated) than making a first optimization for the water retention functions as a not so "optimal" and restrictive

Soil heterogeneity and uncertainty derived from the initial conditions and its implications in the model is not further discussed in the manuscript.

Interactive

comment

\section{Minor comments:}

It is strongly suggested to make a more detailed flow chart of the whole inverse modeling process in a flow chart in which the input and output is clearly showed as figure 2 tells very little information.

Explain clearly the difference between calibration and inverse modeling (the first a general concept and the second one a method for calibration and estimation of input uncertainty) as both terms are used indiscriminately in the abstract, section 3 and conclusions.

In section 4, the expression "A first inversion..." is used but the expression "The second inversion ..." is never found. This is bad English use and makes section 4 very confusing to understand.

Violin Plots (Figures 5 and 8) are not a common and straight forward way to show the results and therefore is suggested to the authors to guide the reader how to interpret the plots. In addition, the mean and confidence intervals are not visible for the modified exponential function.

Reference to Appendix A for equation 1 is missing.

Theta_s is not included in table 1.

Picture of the physical experiment will enhance the paper readability significantly.

Double check the use of commas. 
Interactive comment on Hydrol. Earth Syst. Sci. Discuss., https://doi.org/10.5194/hess-2017336, 2017.

\section{HESSD}

Interactive

comment 